\title{
An International Survey of Applied Face-Matching Training Courses
}

\author{
Reuben Moreton ${ }^{1}$, Catriona Havard ${ }^{1}$, Ailsa Strathie ${ }^{1}, \&$ Graham Pike $^{1}$ \\ ${ }^{1}$ School of Psychology, The Open University, Milton Keynes, UK
}

THIS PAPER HAS NOT BEEN PEER-REVIEWED

\begin{abstract}
Author Note
Reuben Moreton https://orcid.org/0000-0001-6369-6031

Correspondence concerning this article should be addressed to Reuben Moreton, School of Psychology, The Open University, Walton Hall, Milton Keynes, UK, MK7 6AA. Email: reuben.moreton@open.ac.uk
\end{abstract}




\begin{abstract}
Face matching decisions in applied settings, such as policing, forensics and immigration services, are typically carried out by two types of professionals; facial reviewers and facial examiners. Studies have demonstrated large individual differences in the face matching accuracy of both facial reviewers and examiners. Whether variances in training between agencies could be in part responsible for these individual differences is not currently known. Despite the existence of high-level training guidelines produced by the practitioner community the content, duration and delivery of face matching training is not widely understood in the academic research community. The current study aimed to address this gap in the scientific literature and to better understand how different agencies train facial reviewers and facial examiners, using results collected from an international survey. At the group level facial examiners received longer durations of training, covering more topics and with greater inclusion of mentoring than facial reviewers. However, the survey revealed large differences in the duration, delivery methods and content of training by individual agencies at both the facial review and facial examiner level and low inclusion of evidence-based training practices. These results should help researchers to better understand the diversity in training practices and durations of training between different agencies and may help explain the individual differences observed in the performance of face matching professionals in the literature.
\end{abstract}




\section{Introduction}

Face matching is widely used as a means of identification in high stakes, security critical settings, such as law enforcement, forensics, defence and border security. Examples include verifying a passport holders' identity at the border, comparing images of a suspect to CCTV video of an offender or identifying persons of interest in public spaces using automated facial recognition. In applied settings the outcome of a face matching decision could have potentially life changing consequences, such as a person being denied entry at the border, a suspect's arrest in a police investigation or a defendant being convicted and sentenced to imprisonment. For most applied uses of face matching (even in cases where an automated algorithm is used) a human operator is required to act as the final arbiter of the match decision.

In recent years there has been growing interest in the accuracy of trained face matching professionals working in applied settings. White, Towler, \& Kemp (2021) completed a metaanalysis of 12 published studies evaluating the performance of two groups of trained face matching professionals as well as untrained police super recognisers in 25 different experiments. The facial reviewer and facial examiner roles were defined using guidance documents produced by the international Facial Identification Scientific Working Group (FISWG). Facial reviewers are a diverse group of trained professionals, including bank tellers, police officers, border control officers and passport issuance officers, who conduct face matching in high-throughput environments, often to provide investigative and operational leads. Facial reviewers may also work with automated facial recognition systems (Facial Identification Scientific Working Group, 2019b). Facial examiners are specialised face matching professionals, who commonly work in small teams within police, forensic providers and government agencies. Facial examiners conduct face matching by rigorous morphological analysis, comparison and evaluation of images, often in a forensic setting (Facial Identification 
Scientific Working Group, 2019a). The third group, police super recognisers, consisted of face matching professionals selected based on their superior innate ability on various face identification tasks. However, the specific mechanisms by which police super recognisers are recruited and selected in different organisations are not well understood in the literature (White et al., 2021). The results of the facial reviewer and facial examiner are of relevance to the current study.

The meta-analysis showed mixed results between the reviewer and examiner groups. Concerningly, in 12 out of 18 experiments facial reviewers showed no improvement in face matching accuracy over untrained controls and lower performance in half of studies. These findings suggest that the professional experience of the majority of facial reviewers gave no advantage in face matching accuracy, despite this task being a primary part of their role.

In contrast with findings that show that training and professional experience offer no advantage for many facial reviewer groups (e.g. Burton et al., 1999; White, Kemp, Jenkins, Matheson, et al., 2014; Wirth \& Carbon, 2017), professional facial examiners were shown to consistently outperform control groups in all seven tests reviewed in the meta-analysis (see Norell et al., 2015; Phillips et al., 2018; Towler, White, \& Kemp, 2017; White, Dunn, et al., 2015; White, Kemp, Jenkins, Matheson, et al., 2014). Facial examiners often provide expert evidence on facial image comparisons in the courtroom, and undergo lengthy training and mentoring in face matching, employing detailed and rigorous methods to compare the images (Houlton \& Steyn, 2018). As a group, examiners have been shown to perform highly relative to untrained controls in both quick decision face matching tests (White, Phillips, Hahn, Hill, \& O’Toole, 2015) and when allowed to use their own tools and methods (Norell et al., 2015; Phillips et al., 2018). Examiners have also been demonstrated to be more cautious in making comparison decisions particularly from low quality images (Norell et al., 2015), possibly because they are more 
aware of the likely impact that image quality can have, and mindful of the ramifications of an incorrect decision in an applied setting.

It is important to note that forensic facial examiners are not a homogenous group and significant variation in accuracy on tests exists between different examiners. There has also been heavy criticism of certain working practices used by forensic facial examiners, such as the technique of measuring facial proportions (Kleinberg \& Vanezis, 2007; Moreton \& Morley, 2011) and overlaying or superimposing facial images (Strathie \& McNeill, 2016; Strathie, Mcneill, \& White, 2012). For detailed reviews see Edmond, Biber, Kemp, \& Porter, (2008); Mallett \& Evison, (2013) and McNeill, Suchomska, \& Strathie, (2015). Despite past criticisms, the accuracy of examiners at group level has been consistently demonstrated in recent years (Phillips et al., 2018; White, Phillips, et al., 2015). This suggests that further scrutiny of the work of forensic facial examiners is required to establish what it is about this group that gives them their enhanced performance, whether this be training and mentoring, professional expertise, innate perceptual skill or a combination of multiple factors.

One possible explanation for the varied performance of facial reviewers and facial examiners observed in the literature may be the differences in recruitment and training of staff by different agencies. This is particularly pertinent given that an empirical evaluation of short professional face-matching courses found that training durations of less than three days led to no improvements in face-matching accuracy (Towler et al., 2019). Without knowing how training is being delivered by different agencies or whether approaches to training are consistent, it is difficult to identify the factors that contribute to individual differences in face-matching practitioners. This study aimed to review face matching training practices used internationally by agencies that undertake face matching, including policing, forensics and border security, by means of an online survey. The survey asked questions about training practices for two 
different types of face-matching professionals: facial reviewers and facial examiners. The survey questions ascertained who delivered each agency's training, the duration of the training, the topics covered, and how the training was delivered with the objective of determining if there are differences in how training is administered between reviewers and examiners, and if there is consistency in training within the two levels. Any observed differences between training practices for facial reviewers and facial examiners may shed light on the discrepancies in accuracy observed between different professional groups in the literature. The survey also asked whether training practices include elements that have been empirically evidenced to improve face-matching accuracy in the literature, including feedback (Alenezi \& Bindemann, 2013; White, Kemp, Jenkins, \& Burton, 2014), facial feature comparison (Megreya \& Bindemann, 2018; Towler et al., 2017), working in pairs (Dowsett \& Burton, 2015) and testing perceptual skill in face matching (Bobak, Dowsett, \& Bate, 2016).

\section{Methods}

\section{Participants}

The sample consisted of 24 international agencies that undertook face matching in an applied setting, including police forces, forensic providers and immigration services at the time the survey was administered. The sample included agencies from 12 different countries, shown in Figure 1. Nine of the agencies were based in Europe (38\%), eight in North America \& Canada (33\%), five in Australia (20\%) and two in the Middle East (6\%). 


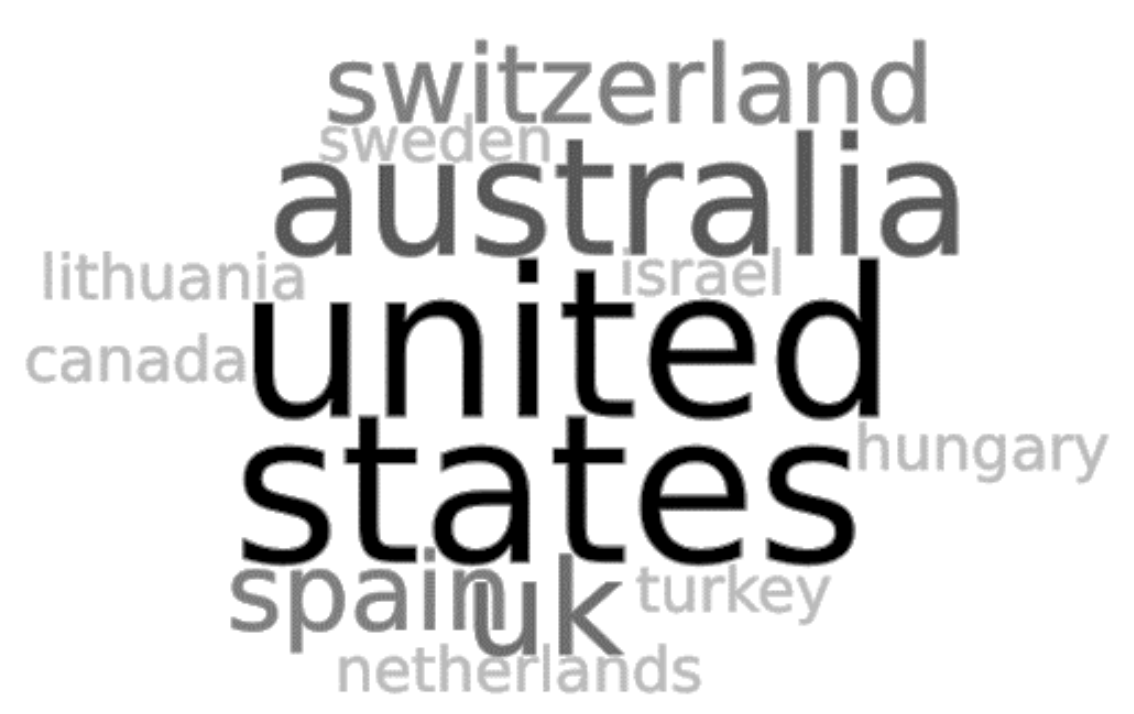

Figure 1 - Word cloud of participating countries

Participants were recruited via email using the mailing lists of face-matching practitioner working groups. Those contacted were requested to respond on behalf of their organisation or department rather than as an individual, to prevent duplicated responses from different individuals working for the same agency. Results were collected between September and December 2017. Of the agencies surveyed 15 trained facial reviewers (62\%) and 18 trained facial examiners $(75 \%)(*$ note some agencies provide face matching training at multiple levels).

\section{Procedure}

Participants were provided with a link to the survey via email, which was hosted on the Qualtrics platform. Upon starting the survey, participants were presented with a body of text providing some background information, confirmation of consent and the aims of the survey, including the following: 
"The aim of the survey is to find out more about current practices in face-matching training in applied settings (e.g. policing, forensics, security and border control). The survey will ask questions on the type of training provided by your agency, what specific topics are covered by the training and how the training is delivered. The results of this survey will be used to direct future research into effective training strategies in facial identification and the production of good practice guidance and standards in the field."

Participants were then requested to provide the country in which their agency was based and, optionally, to provide their agency name and department to prevent duplication of agencies (all data has since been anonymised). The next page required participants to provide information about the types of face matching their agency conducted; facial review and/or facial examination and what types of material they receive for comparison. The remainder of the survey focussed upon face-matching training practices, specifically who delivered training, how training was delivered, the duration of training and the content of training material. The study received a favourable opinion from the ethical committee of the Open University. Results from the survey were analysed in SPSS. 


\section{Results and discussion}

A summary overview of the results from the survey by agency is provided in Table 1, including type of training, method of training delivery and duration of training. The subsections below provide a detailed breakdown of responses to the survey, including statistical analyses of results between the reviewer training $(n=15)$ and examiner training $(n=18)$ groups and a discussion of the results per subsection. 
Table 1 - Overview of training type, delivery method and duration by agency

\begin{tabular}{|c|c|c|c|c|c|c|c|}
\hline \multirow[b]{2}{*}{ Agency } & \multirow[b]{2}{*}{ Type of training } & \multicolumn{5}{|c|}{ Delivery method } & \multirow[b]{2}{*}{ Duration of training } \\
\hline & & $\stackrel{\mathscr{\Xi}}{\Xi}$ & 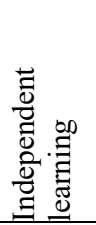 & 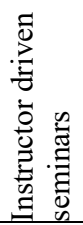 & 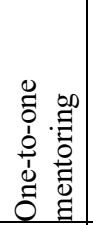 & $\begin{array}{l}\bar{\Xi} \\
\overline{0}\end{array}$ & \\
\hline 1 & Reviewer & $\mathrm{x}$ & $\mathrm{x}$ & & & & $<1$ day \\
\hline 2 & Reviewer & & $\mathrm{x}$ & & $\mathrm{x}$ & & $1-6$ months \\
\hline 3 & Reviewer & & & $\mathrm{x}$ & & & $<1$ day \\
\hline 4 & Reviewer & & & $\mathrm{x}$ & & & 1 day \\
\hline 5 & Reviewer & & & $x$ & & & $<1$ day \\
\hline 6 & Reviewer & $\mathrm{x}$ & & $\mathrm{x}$ & & & $<1$ day \\
\hline \multirow{2}{*}{7} & Reviewer & & $\mathrm{x}$ & $\mathrm{x}$ & $\mathrm{x}$ & & $1-6$ months \\
\hline & Examiner & & $\mathrm{x}$ & $\mathrm{x}$ & $\mathrm{x}$ & & $1-5$ years \\
\hline \multirow{2}{*}{8} & Reviewer & & & $\mathrm{x}$ & $x$ & & 2-4 weeks \\
\hline & Examiner & & $\mathrm{x}$ & $\mathrm{x}$ & $\mathrm{x}$ & $\mathrm{x}$ & $5+$ years \\
\hline \multirow{2}{*}{9} & Reviewer & $\mathrm{x}$ & $x$ & $\mathrm{x}$ & $\mathrm{x}$ & & $1-6$ months \\
\hline & Examiner & $\mathrm{x}$ & $\mathrm{x}$ & $\mathrm{x}$ & $\mathrm{x}$ & & $1-6$ months \\
\hline \multirow{2}{*}{10} & Reviewer & & & $\mathrm{x}$ & $\mathrm{x}$ & & $2-4$ weeks \\
\hline & Examiner & & $x$ & $\mathrm{x}$ & $\mathrm{x}$ & & $1-5$ years \\
\hline \multirow{2}{*}{11} & Reviewer & $\mathrm{x}$ & $\mathrm{x}$ & $\mathrm{x}$ & $\mathrm{x}$ & & $1-6$ months \\
\hline & Examiner & $\mathrm{x}$ & $x$ & $x$ & $\mathrm{x}$ & & $1-6$ months \\
\hline \multirow{2}{*}{12} & Reviewer & & & $\mathrm{x}$ & $\mathrm{x}$ & & $1-6$ months \\
\hline & Examiner & & & $\mathrm{x}$ & $x$ & & $1-6$ months \\
\hline \multirow{2}{*}{13} & Reviewer & & & $x$ & & & $2-5$ days \\
\hline & Examiner & & $\mathrm{x}$ & $\mathrm{x}$ & $\mathrm{x}$ & & $1-6$ months \\
\hline \multirow{2}{*}{14} & Reviewer & $x$ & $x$ & & $x$ & & $1-6$ months \\
\hline & Examiner & $\mathrm{x}$ & $\mathrm{x}$ & & $\mathrm{x}$ & & $1-5$ years \\
\hline \multirow{2}{*}{15} & Reviewer & $\mathrm{x}$ & $\mathrm{x}$ & & $\mathrm{x}$ & & 6-12 months \\
\hline & Examiner & $x$ & $x$ & $x$ & $\mathrm{x}$ & & $1-5$ years \\
\hline 16 & Examiner & & $\mathrm{x}$ & $\mathrm{x}$ & $\mathrm{x}$ & & $1-5$ years \\
\hline 17 & Examiner & & & $\mathrm{x}$ & & & 2-4 weeks \\
\hline 18 & Examiner & & $\mathrm{x}$ & & $\mathrm{x}$ & & $1-5$ years \\
\hline 19 & Examiner & & $x$ & $\mathrm{x}$ & $x$ & & $5+$ years \\
\hline 20 & Examiner & & & $x$ & $\mathrm{x}$ & & 6-12 months \\
\hline 21 & Examiner & & & $\mathrm{x}$ & & & No answer \\
\hline 22 & Examiner & & & & $\mathrm{x}$ & & $1-5$ years \\
\hline 23 & Examiner & & $x$ & $\mathrm{x}$ & $\mathrm{x}$ & $x$ & $1-5$ years \\
\hline 24 & Examiner & & & & $\mathrm{x}$ & & $1-5$ years \\
\hline
\end{tabular}




\section{Training delivery results}

Of the 24 agencies surveyed, almost $80 \%$ delivered training in face matching for facial reviewers and facial examiners internally. One third received training from external agencies and only a quarter procured training from commercial providers (Table 2).

Table 2 - Source of training by agency

\begin{tabular}{lcl}
\hline Source of Training & Agency (n=24) \\
\hline Internally within your agency & $79.2 \%$ & $(19)$ \\
Externally from another agency & $33.3 \%$ & $(8)$ \\
Supply training to other agencies & $25.0 \%$ & $(6)$ \\
Externally from a commercial provider & $25.0 \%$ & $(6)$ \\
Other & $12.5 \%$ & $(3)$ \\
\hline
\end{tabular}

Table 3 shows the methods of training delivery for reviewer training and examiner training. Online training was the least common delivery method for both reviewer and examiner training but was almost twice as common for reviewer training. One-to-one mentoring was used in almost $90 \%$ of examiner training but only $60 \%$ of reviewer training. Instructor driven seminars were common in both examiner and reviewer training. Independent learning featured more prominently in examiner training but in less than half of reviewer training. Differences in delivery method between reviewer and examiner training delivery methods were not found to be significant at the $95 \%$ confidence level.

Table 3 - Delivery methods for reviewer and examiner training

\begin{tabular}{lclcl}
\hline Delivery Method & Reviewer Training (n=15) & Examiner Training (n=18) \\
\hline Online training & $40.0 \%$ & $(6)$ & $22.2 \%$ & $(4)$ \\
Independent learning & $46.7 \%$ & $(7)$ & $66.7 \%$ & $(12)$ \\
Instructor driven seminars & $73.3 \%$ & $(11)$ & $77.8 \%$ & $(14)$ \\
One-to-one mentoring & $60.0 \%$ & $(9)$ & $88.9 \%$ & $(16)$ \\
Other & $0.0 \%$ & $(0)$ & $11.1 \%$ & $(2)$ \\
\hline
\end{tabular}




\section{Training delivery discussion}

Online training was the least common method of training delivery in the sample. There were also no agencies in the sample that used only online training delivery (see Table 1). This is a promising finding as a study of the efficacy of online face-matching training found that short durations of online training (less than three days) do not improve face-matching accuracy (Towler et al., 2019). Instructor driven seminars were the most common delivery method for reviewer training and second most common for examiner training, with six agencies only using instructor driven training. Woodhead et al. (1979) found no improvement in trainee comparison accuracy after a three-day instructor driven face-matching course and Towler et al. (2019) found no improvements after a one-day course and only limited and inconsistent improvements after a three-day course. These results suggest that agencies should opt against only using instructor-driven seminars to deliver training, particularly if that training is of short duration.

Almost all of the agencies providing examiner training used some form of one-to-one mentoring $(88.9 \%)$. One-to-one mentoring was the second most common delivery method for reviewer training, used by $60 \%$ of agencies. Mentoring can be used as a means of transferring tacit knowledge to trainees, as often tacit knowledge is not formally recorded and is instead based on the experiences of more senior colleagues (Mayfield, 2010). Mentoring has been found to be highly effective in the training of new teachers (Langdon, 2014) and in transferring knowledge and experience in corporate business (Sosik, Godshalk, \& Yammarino, 2004). Despite being the most common delivery method for facial examiner training in this survey the author is not aware of any study to date that has evaluated the effectiveness of mentorship for improving face-matching accuracy. Dowsett \& Burton, (2015) found that when novices worked in pairs on a face-matching task the higher performing individuals in the pair provided a learning effect for lower performing individuals, which transferred to a second face-matching 
task completed individually. But this design is unlikely to be representative of a professional face-matching mentorship programme. Given the widespread use of one-to-one mentoring in face-matching training reported in this survey, future research should look at how mentoring is being delivered by face-matching agencies and establish the effectiveness of this approach in improving face-matching accuracy.

Studies to date have only looked at one type of delivery method for face-matching training over short durations (Towler et al., 2019; Woodhead et al., 1979). It is important to note that the majority of agencies surveyed used multiple training delivery methods for both reviewers and examiners (see Table 1), but there was little consistency in the delivery methods used between different agencies. When deciding how to deliver training face-matching trainers should consider the research that has already been conducted on online and short instructordriven delivery methods. There also needs to be further work with researchers to establish what combinations of delivery methods are most effective. This collaborative approach will provide a drive for consistency in training delivery that is much needed based on the findings of the survey presented here.

\section{Training duration results}

Table 4 presents a comparison of survey results for training duration between the reviewer and examiner training groups. There are clear differences in training duration between the groups. Examiner training was generally of longer duration, with $66.7 \%$ of agencies delivering training that lasted a year or more in duration and only one agency (5.6\% of the sample) delivered examiner training that was less than a month in duration. Conversely, of the agencies surveyed, no reviewer training exceeded 12 months in duration and 40\% of agency reviewer training was five days or less. A Mann-Whitney $U$ test revealed that the distribution of training duration was 
significantly different between examiner and reviewer training groups $(U=20.5, \mathrm{p}=.001)$, with reviewer training tending to be shorter than examiner training

Table 4-Duration of reviewer and examiner training

\begin{tabular}{lrrrr}
\hline Duration & Reviewer Training $(\mathbf{n}=\mathbf{1 5})$ & Examiner Training (n=18) \\
\hline Less than 1 day & $26.7 \%$ & $(4)$ & $0.0 \%$ & $(0)$ \\
1 day & $6.7 \%$ & $(1)$ & $0.0 \%$ & $(0)$ \\
2 to 5 days & $6.7 \%$ & $(1)$ & $0.0 \%$ & $(0)$ \\
2 to 4 weeks & $13.3 \%$ & $(2)$ & $5.6 \%$ & $(1)$ \\
1 to 6 months & $40.0 \%$ & $(6)$ & $16.7 \%$ & $(3)$ \\
6 to 12 months & $6.7 \%$ & $(1)$ & $5.6 \%$ & $(1)$ \\
1 to 5 years & $0.0 \%$ & $(0)$ & $55.6 \%$ & $(10)$ \\
5 + years & $0.0 \%$ & $(0)$ & $11.1 \%$ & $(2)$ \\
\hline
\end{tabular}

Figure 2 shows the distributions of training duration for both examiner and reviewer training. Examiner training is clearly skewed towards longer durations (one year and greater). However, there is a wide range in training duration from two to four weeks to five plus years. Reviewer training durations show a bimodal distribution with $40 \%$ of agencies providing one to six months of training and $26.7 \%$ of agencies providing less than one day of training. As for examiner training there was a very wide range in reviewer training duration. 


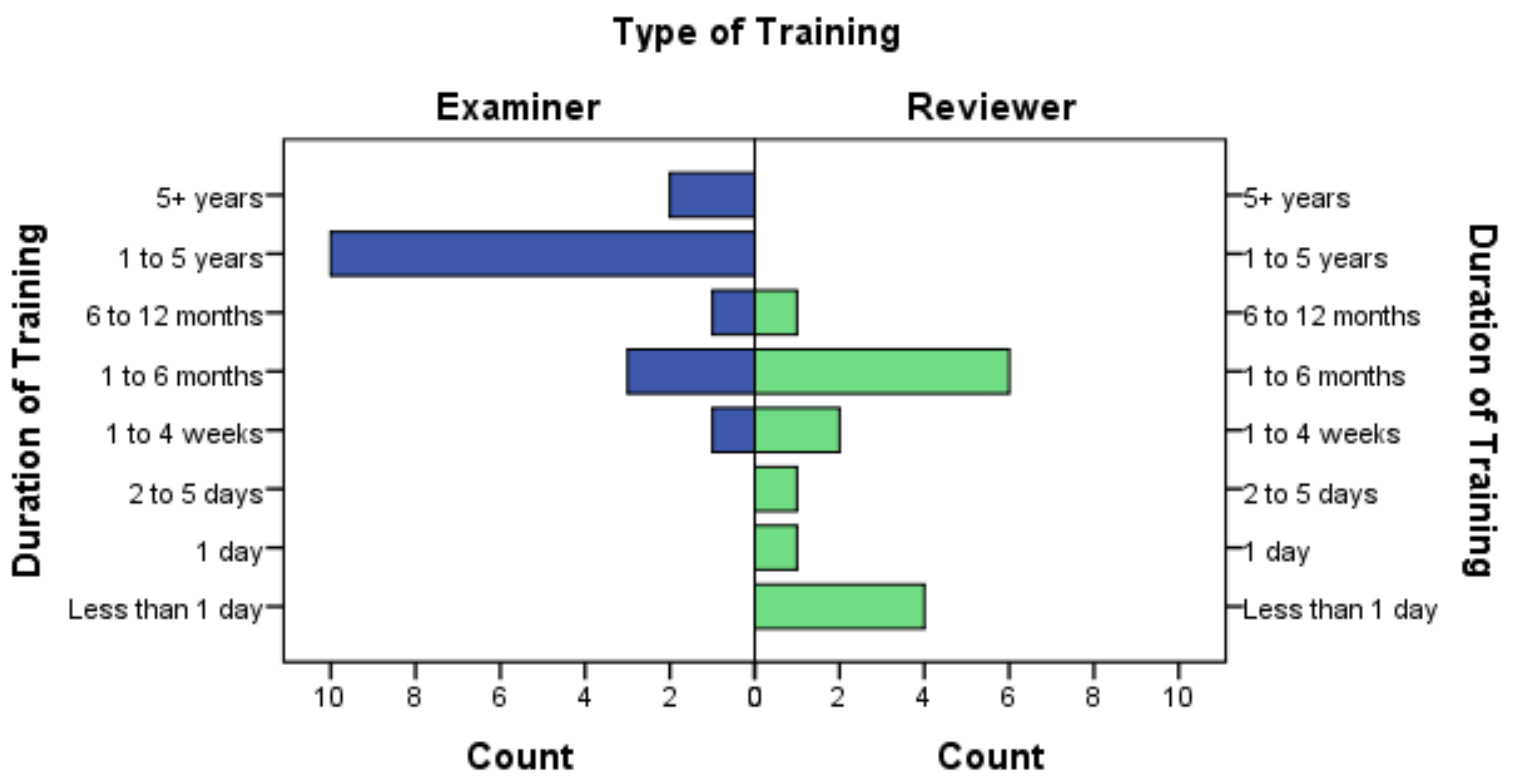

Figure 2 - Frequency distributions for durations of examiner and reviewer training

\section{Training duration discussion}

It is clear that as a group examiners receive much longer durations of training than most reviewers and this may offer a possible explanation for the greater accuracy of trained examiners over trained reviewers observed in some studies (Phillips et al., 2018; White, Dunn, et al., 2015; White, Phillips, et al., 2015). However, examiners have shown significant individual variability in face matching on both quick decision tests and more challenging comparisons that resemble operational imagery (Norell et al., 2015; Phillips et al., 2018; White, Phillips, et al., 2015). Like the wide range in training delivery methods, training duration is comparably inconsistent across different agencies. For examiners, the majority of agencies provided training for one to five years, whereas the second highest count for training duration was one to six months. At the lower bounds of these training durations that is a difference factor of 12 (one year compared to one month) and the shortest durations of examiner training demonstrated from this survey is only two to four weeks. Given these substantial differences in time it can be assumed that training practices for examiners are very different between 
agencies. If training does have a significant bearing on examiner accuracy, then the variability in training duration may contribute to the individual differences between examiners observed in the literature.

Individual facial reviewer accuracy also varies substantially in the literature, with some reviewers performing at examiner group levels (Phillips et al., 2018) and others at the level of untrained novices or even chance (White, Kemp, Jenkins, Matheson, et al., 2014). The diversity in training durations for reviewers is equally, if not more, concerning than that of examiners. $40 \%$ of agencies provided lone to six months of training but another $40 \%$ provided reviewer training that was five days or less and $26 \%$ of agencies provided less than one days training. This is an alarming finding given that studies have shown limited or no improvements in accuracy from three-day training courses and no improvements from training courses that are one day or less (Towler et al., 2019; Woodhead et al., 1979). It can only be assumed that reviewers being trained for one to six months are receiving a very different training experience to those who are trained for less than a day.

Although job-specific requirements will likely vary between agencies, the tasks and responsibilities of face-matching personnel from different agencies should be broadly comparable. That different agencies are so inconsistent in the durations of training for both examiners and reviewers is a critical issue that should be addressed as a priority. Communication and collaborative working between agencies should be the first step, which could be facilitated by practitioner working groups such as FISWG and ENFSI. Interestingly, surveyed agencies that provide training to both reviewers and examiners have longer durations of reviewer training than those that only train reviewers (see Table 1). This suggests there may be some overlap between reviewer and examiner training practices for agencies that do both. 
Current research has only addressed very short durations of training (maximum three days), but the results of the current survey show that some training lasts for far longer than this, suggesting that further research should look at effectiveness of longer-term training. When testing examiner and reviewer accuracy on face-matching tasks researchers should also establish the extent of training received by participants to determine if there is a relationship between training duration and accuracy.

\section{Training topic results}

The survey asked respondents whether training included training topics recommended by international best practice documents (European Network of Forensic Science Institutes, 2018; Facial Identification Scientific Working Group, 2010). Table 5 shows how many agencies covered each general topic. There was an overall trend for examiner training to cover more of the recommended topics than reviewer training. The only topic to be covered by all agencies was methods of comparison and this was for examiner training. None of the differences between examiner and reviewer training were significant at the $95 \%$ confidence level.

Table 5 - Training topics covered by examiner and reviewer training

\begin{tabular}{lcc}
\hline Training Topics & Reviewer training (n=15) & Examiner training (n=18) \\
\hline Anatomy & $66.7 \%(10)$ & $94.4 \%(17)$ \\
Image science & $66.7 \%(10)$ & $77.8 \%(14)$ \\
Image processing & $66.7 \%(10)$ & $83.3 \%(15)$ \\
Comparison methods & $80.0 \%(12)$ & $100 \%(18)$ \\
\hline
\end{tabular}

The following sections breakdown the four recommended training topics by subtopic based on best practice documentation, with statistical analyses of results between examiner training and reviewer training using odds ratios and Fisher's exact test of significance at the $95 \%$ confidence level. 
Anatomy training subtopic results

The examiner training group covered a high proportion of subtopics in facial anatomy, ranging from $94 \%$ of agencies covering 'face shape', 'features of the skin' and 'creases and lines' to $72 \%$ covering 'juvenile development' and 'alterations to the face'. Anatomy training was covered in less detail by reviewer training, ranging from $66.7 \%$ of agencies covering features of the 'nose', 'mouth', 'features of the skin', 'creases and lines' and 'facial expression' to only $27 \%$ of agencies providing training in 'juvenile development' (Table 6)

Table 6-Anatomy training subtopics covered by reviewer and examiner training

\begin{tabular}{lccccc}
\hline Anatomy Subtopics & $\begin{array}{c}\text { Reviewer Training } \\
(\mathbf{n}=\mathbf{1 5})\end{array}$ & $\begin{array}{c}\text { Examiner Training } \\
(\mathbf{n}=\mathbf{1 8})\end{array}$ \\
\hline Face shape & $60.0 \%$ & $(9)$ & $94.4 \%$ & $(17)$ \\
Eyes & $60.0 \%$ & $(9)$ & $83.3 \%$ & $(15)$ \\
Ears & $60.0 \%$ & $(9)$ & $88.9 \%$ & $(16)$ \\
Nose & $66.7 \%$ & $(10)$ & $88.9 \%$ & $(16)$ \\
Mouth & $66.7 \%$ & $(10)$ & $88.9 \%$ & $(16)$ \\
Chin and jaw & $60.0 \%$ & $(9)$ & $88.9 \%$ & $(16)$ \\
Features of the skin (e.g. scars/marks) & $66.7 \%$ & $(10)$ & $94.4 \%$ & $(17)$ \\
Bones of the skull & $46.7 \%$ & $(7)$ & $77.8 \%$ & $(14)$ \\
Muscles of the face* & $33.3 \%$ & $(5)$ & $77.8 \%$ & $(14)$ \\
Creases and lines & $66.7 \%$ & $(10)$ & $94.4 \%$ & $(17)$ \\
Facial Expression & $66.7 \%$ & $(10)$ & $83.3 \%$ & $(15)$ \\
Effects of ageing* & $46.7 \%$ & $(7)$ & $88.9 \%$ & $(16)$ \\
Juvenile development* & $26.7 \%$ & $(4)$ & $72.2 \%$ & $(13)$ \\
Permanence of features* & $40.0 \%$ & $(6)$ & $88.9 \%$ & $(16)$ \\
Alterations to the face (e.g. piercings) & $53.3 \%$ & $(8)$ & $72.2 \%$ & $(13)$ \\
\hline
\end{tabular}

Statistically significant differences were observed between reviewer and examiner training for the following anatomy subtopics; 'muscles of the face' $(\mathrm{OR}=9.6,95 \% \mathrm{CI}: 1.9,47.4, p=0.005$, Fisher's exact test), 'effects of ageing' (OR $=9.1,95 \% \mathrm{CI}: 1.5,54.5, p=0.020$, Fisher's exact test), 'juvenile development' ( $\mathrm{OR}=7.1,95 \% \mathrm{CI}: 1.5,33.3, p=0.015$, Fisher's exact test $)$ and 'permanence of features' $(\mathrm{OR}=9.1,95 \% \mathrm{CI}: 1.5,54.5, p=0.020$, Fisher's exact test $)$.

All anatomy subtopics were more frequently covered in examiner training than in reviewer training, however most of these differences were not statistically significant. The lack of 
significance observed for certain differences between reviewer training and examiner training may be attributable to the small size of the sample in the study. For example, although it is more than seven times more likely for examiner training to cover both 'face shape' and 'features of the skin' (e.g. scars and marks) than the reviewer training group, this was not found to be significant at the $95 \%$ confidence level $(\mathrm{OR}=8.5,95 \% \mathrm{CI}: 0.87,83.5, p=0.070$, Fisher's exact test). A post hoc power analysis of Face Shape revealed a 1 - $\beta$ error probability of 0.67 $(\alpha=0.05)$ for Fisher's exact test, indicating the sample may be of insufficient size to reject the null hypothesis even when a large effect is present (i.e. a propagation of type II errors). The lack of statistical power is likely to also be an issue for other training subtopics, not just in facial anatomy. Although only a minority of results are significant at the $95 \%$ confidence level there is a clear trend for examiner training covering proportionally more anatomy topics than reviewer training.

Image science and processing training subtopic results

Table 7 shows the proportion of agencies that covered image science subtopics broken down by training type (reviewer or examiner), Table 8 shows these results for image processing subtopics.

Table 7 - Image science subtopics covered by reviewer and examiner training

\begin{tabular}{lcccc}
\hline Image Science Subtopics & $\begin{array}{c}\text { Reviewer Training } \\
(\mathbf{n}=\mathbf{1 5})\end{array}$ & $\begin{array}{c}\text { Examiner Training } \\
(\mathbf{n}=\mathbf{1 8})\end{array}$ \\
\hline Properties of visible light & $40.0 \%$ & $(6)$ & $66.7 \%$ & $(12)$ \\
Properties of non-visible wavelengths & $33.3 \%$ & $(5)$ & $55.6 \%$ & $(10)$ \\
Image capture and camera sensors & $33.3 \%$ & $(5)$ & $61.1 \%$ & $(11)$ \\
Impact of lighting and camera exposure & $66.7 \%$ & $(10)$ & $77.8 \%$ & $(14)$ \\
Geometric distortions & $60.0 \%$ & $(9)$ & $77.8 \%$ & $(14)$ \\
Aspect ratio distortion & $53.3 \%$ & $(8)$ & $77.8 \%$ & $(14)$ \\
Pixel resolution & $66.7 \%$ & $(10)$ & $77.8 \%$ & $(14)$ \\
Image compression & $66.7 \%$ & $(10)$ & $77.8 \%$ & $(14)$ \\
Video compression & $33.3 \%$ & $(5)$ & $66.7 \%$ & $(12)$ \\
\hline
\end{tabular}


Table 8 - Image processing subtopics covered by reviewer and examiner training

\begin{tabular}{lcccc}
\hline Image Processing Subtopics & $\begin{array}{c}\text { Reviewer Training } \\
(\mathbf{n}=\mathbf{1 5})\end{array}$ & $\begin{array}{c}\text { Examiner Training } \\
(\mathbf{n}=\mathbf{1 8})\end{array}$ \\
\hline Brightness and contrast adjustments & $73.3 \%$ & $(11)$ & $83.3 \%$ & $(15)$ \\
Rotations and cropping & $66.7 \%$ & $(10)$ & $83.3 \%$ & $(15)$ \\
Sharpening and blurring & $53.3 \%$ & $(8)$ & $77.8 \%$ & $(14)$ \\
Scaling & $60.0 \%$ & $(9)$ & $83.3 \%$ & $(15)$ \\
Colour channel separation & $46.7 \%$ & $(7)$ & $66.7 \%$ & $(12)$ \\
Effects on facial appearance & $60.0 \%$ & $(9)$ & $83.3 \%$ & $(15)$ \\
\hline
\end{tabular}

All subtopics in image processing and image science were covered by more examiner training agencies than reviewer training agencies, however none of these differences were found to be statistically significant at the $95 \%$ confidence level, which may be due to a lack of statistical power as mentioned above. In general, fewer agencies covered image science subtopics than image processing subtopics for both reviewer and examiner training.

Comparison method subtopics results

The proportions of reviewer and examiner training that covered comparison method subtopics are shown in Table 9. Almost all subtopics follow the previous pattern of being covered in a higher proportion of examiner training than reviewer training. 'Use of automated facial recognitions systems' does not follow this trend and is covered by a slightly higher proportion of reviewer training agencies than examiners (73.3\% and $61.1 \%$ respectively), however this difference was not statistically significant at the $95 \%$ confidence level. 
Table 9 - Comparison method subtopics covered by reviewer and examiner training

\begin{tabular}{lclrl}
\hline Comparison Method Subtopics & $\begin{array}{c}\text { Reviewer Training } \\
(\mathbf{n = 1 5})\end{array}$ & $\begin{array}{c}\text { Examiner Training } \\
(\mathbf{n}=\mathbf{1 8})\end{array}$ \\
\hline Instruction in the ACE-V framework* & $53.3 \%$ & 8 & $100.0 \%$ & 18 \\
Instruction in holistic comparison & $66.7 \%$ & 10 & $66.7 \%$ & 12 \\
Limitations of holistic comparison & $66.7 \%$ & 10 & $83.3 \%$ & 15 \\
Instruction in morphological comparison & $80.0 \%$ & 12 & $100.0 \%$ & 18 \\
Limitations of morphological comparison* & $53.3 \%$ & 8 & $88.9 \%$ & 16 \\
Instruction in facial feature classification & $40.0 \%$ & 6 & $55.6 \%$ & 10 \\
Limitations of facial feature classification & $53.3 \%$ & 8 & $83.3 \%$ & 15 \\
Instruction in photo anthropometry & $26.7 \%$ & 4 & $50.0 \%$ & 9 \\
Limitations of photo anthropometry* & $46.7 \%$ & 7 & $88.9 \%$ & 16 \\
Instruction in superimposition & $20.0 \%$ & 3 & $50.0 \%$ & 9 \\
Limitations of superimposition* & $46.7 \%$ & 7 & $88.9 \%$ & 16 \\
Use of automated facial recognition & $73.3 \%$ & 11 & $61.1 \%$ & 11 \\
Human facial recognition & $73.3 \%$ & 11 & $77.8 \%$ & 14 \\
Cognitive bias & $66.7 \%$ & 10 & $83.3 \%$ & 15 \\
Own-race effects & $53.3 \%$ & 8 & $61.1 \%$ & 11 \\
Evaluating comparison findings & $60.0 \%$ & 9 & $83.3 \%$ & 15 \\
Peer-review and independent verification & $66.7 \%$ & 10 & $83.3 \%$ & 15 \\
\hline
\end{tabular}

Statistically significant differences were observed between reviewer and examiner training for the following methods of comparison subtopics; 'ACE-V framework' (OR $=15.7,95 \% \mathrm{CI}$ : 1.6, 150.1, $p=0.011$, Fisher's exact test), 'limitations of morphological comparison' ( $\mathrm{OR}=7$, 95\% CI: 1.2, 41.8, $p=0.047$, Fisher's exact test), 'limitations of photo anthropometry' (OR = 9.1, 95\% CI: $1.5,54.5, p=0.020$, Fisher's exact test) and 'limitations of superimposition' (OR $=9.1,95 \% \mathrm{CI}: 1.5,54.5, p=0.020$, Fisher's exact test). 'Instruction in the ACE-V framework' and 'instruction in morphological comparison are the only subtopics from the survey to be covered by all $100 \%$ of examiner training agencies. ACE-V is an acronym for analyse, compare, evaluate-verify a framework used in several forensic comparison disciplines, such as fingerprint comparison (European Network of Forensic Science Institutes, 2015). 


\section{Training topics discussion}

All training subtopics were covered by a higher proportion of examiner training agencies than reviewer agencies, with the exception of 'instruction in the use of automated facial recognition systems'. Given the longer durations of training for examiners it is not surprising that more subtopics are included in their training, and as facial reviewers are more likely to use automated facial recognition systems, it is also unsurprising that this topic is more commonly covered in reviewer rather than examiner training.

For anatomy training there were statistically significant differences observed for four subtopics ('muscles of the face', 'effects of ageing', 'juvenile development' and 'permanence of features'). These four topics appear to be the more detailed and complex aspects of facial anatomy, supporting the notion that examiner training is more in-depth than reviewer training. For image science and image processing, examiner training agencies covered proportionally more subtopics, although no differences were found to be statistically significant.

Anatomy and imaging topics were covered by a high proportion of training agencies (see Table 5) as recommended by best practice and are explored in more detail for examiner training. However, at present research demonstrates that knowledge of these topics does not improve face-matching accuracy (Towler, 2016). Therefore, it is unlikely that more in-depth training in anatomy and imaging is responsible for the enhanced accuracy of examiners, though such training may provide useful knowledge for explaining findings and observations, particularly in reports and during testimony in court. Greater inclusion of image science topics in examiner training may be a contributing factor to why examiners are more cautious than novices when comparing low quality images (Norell et al., 2015).

For the methods of comparison subtopics, four statistically significant differences were observed between reviewer and examiner training agencies; 'instructions in the ACE-V 
framework', 'limitations of morphological comparison', 'limitations of photo anthropometry and 'limitations of superimposition'. These differences indicate that examiner training may be more method orientated than reviewer training with a greater emphasis on the associated limitations of different comparison methods. This knowledge may also be contributing to why examiners are generally more cautious when making comparisons than novices.

It is very concerning indeed that half of examiner training agencies were providing instruction in the use of facial feature classification, photo anthropometry and superimposition, despite these methods being demonstrated to be unreliable in the literature (Kleinberg \& Vanezis, 2007; Moreton \& Morley, 2011; Ritz-Timme et al., 2011; Strathie \& McNeill, 2016; Strathie et al., 2012; Towler, White, \& Kemp, 2014) and best practice guidance recommending the methods not be used in comparison (European Network of Forensic Science Institutes, 2018; Facial Identification Scientific Working Group, 2012). It is highly inadvisable for agencies to instruct trainees in methods that have been repeatably demonstrated to be unreliable.

Methods of comparison also included the only two subtopics from the survey covered by $100 \%$ of agencies, and this was only for examiner training. These subtopics were 'instruction in the ACE-V framework' and 'instruction in morphological comparison'. ACE-V is a sequential process used in forensic comparison disciplines whereby the suspect material (e.g. CCTV video) is first analysed in isolation to determine its suitability for comparison, then compared to the reference material (e.g. a custody mugshot) and the findings from the comparison are evaluated to determine the weight of evidence. Then the process is independently verified by another examiner who repeats the process (European Network of Forensic Science Institutes, 2018). That this subtopic is covered by $100 \%$ of examiner training and only half $(53 \%)$ of reviewer training supports that examiners are more likely to conduct rigorous and detailed face- 
matching examinations whereas reviewers often work in high throughput environments where time limits are more constrained (Facial Identification Scientific Working Group, 2010).

'Instruction in morphological comparison' was also covered by $100 \%$ of examiner training agencies. Morphological comparison refers to the process of comparing faces on a feature-byfeature basis rather than holistically (Facial Identification Scientific Working Group, 2012). Morphological comparison has been demonstrated to improve the accuracy of novices on facematching tasks when the images are a match (Megreya \& Bindemann, 2018; Towler et al., 2017). The fact that all examiner training reviewed as part of this survey included both instruction in ACE-V and instruction in morphological comparison indicate that forensic facial examiners compare faces in the qualitatively different way to novices (Towler et al., 2017). By being trained in comparing faces in a sequential series of processes on a feature-by-feature basis, examiners may be learning to override the intuitive, quick decision comparisons used by untrained novices and instead learn to make comparisons in a more systematic but resource intensive and time-consuming way.

\section{Evidence-based training strategies results}

The previous sections from the survey focussed on training topics recommended by facematching practitioner working groups. This section of the survey asked respondents whether their face-matching training programmes included any evidence-based exercises, which have been demonstrated to improve face-matching ability in the literature. Table 10 shows the evidence-based approaches to training included in the survey with supporting references. 
Table 10 - Evidence-based approaches to improving face-matching accuracy

\section{Evidence-based approach}

1. Face-matching exercises

2. Feedback on comparison responses

3. Comparison of specific facial features

4. Working on tasks in pairs or groups

5. Face matching using a facial feature list

6. Enhancement of images for comparison (e.g. blurring pixelated images)

7. Testing perceptual skill prior to training

8. Testing perceptual skill after training

\section{Reference}

White et al., 2014. Feedback training for facial image comparison

Megreya \& Bindemann, 2018. Feature instructions improve face-matching accuracy

Dowsett \& Burton, 2015. Unfamiliar face matching: Pairs outperform individuals and provide a route to training

Towler, White, et al., 2017. Evaluating the feature comparison strategy for forensic face identification

Noyes, 2016. Face recognition in challenging situations

Bobak, Dowsett, \& Bate, 2016. Solving the border problem: Evidence of enhanced face matching in individuals with extraordinary face recognition skills

Table 11 shows the number of agencies that included evidence-based approaches in reviewer and examiner training. The majority of evidence-based approaches followed the trend of other topics in the survey by being more widely adopted in examiner training. Testing face-matching ability before and after training, however, were both more common in reviewer training courses. Evidence-based approaches have a very wide range in adoption from $27 \%$ for working in pairs or groups on face-matching tasks, up to $94 \%$ for inclusion of face-matching exercises. The only significant difference observed between reviewer and examiner training was in the use of facial feature checklists in face matching ( $\mathrm{OR}=7,95 \% \mathrm{CI}: 1.2,41.8, p=0.047$, Fisher's exact test). 
Table 11 -Evidence-based approaches used in reviewer and examiner training

\begin{tabular}{lcccl}
\hline Research-based approach & $\begin{array}{c}\text { Reviewer Training } \\
(\mathbf{n}=\mathbf{1 5})\end{array}$ & $\begin{array}{c}\text { Examiner Training } \\
(\mathbf{n}=\mathbf{1 8})\end{array}$ \\
\hline Face-matching exercises & $93.3 \%$ & $(14)$ & $94.4 \%$ & $(17)$ \\
Feedback on comparison responses & $66.7 \%$ & $(10)$ & $88.9 \%$ & $(16)$ \\
Comparison of specific facial features & $60.0 \%$ & $(9)$ & $83.3 \%$ & $(15)$ \\
Working on tasks in pairs or groups & $26.7 \%$ & $(4)$ & $33.3 \%$ & $(6)$ \\
Face matching using a facial feature list* & $46.7 \%$ & $(7)$ & $88.9 \%$ & $(16)$ \\
Enhancement of images for comparison & $60.0 \%$ & $(9)$ & $83.3 \%$ & $(15)$ \\
Testing perceptual skill prior to training & $40.0 \%$ & $(6)$ & $38.9 \%$ & $(7)$ \\
Testing perceptual skill after training & $60.0 \%$ & $(9)$ & $55.6 \%$ & $(10)$ \\
\hline
\end{tabular}

\section{Evidence-based training strategies discussion}

Individual differences in face matching have been consistently demonstrated in the facematching literature, for many years (see Lander et al., 2018). However, only $40 \%$ of agencies tested personnel prior to enrolment in training. Surprisingly, more agencies tested facematching ability after training (up to 60\%). Researchers have advocated pre-screening of facematching accuracy as a means to identify high performers for recruitment into operational facematching roles (Bobak et al., 2016; White et al., 2021). This is of particular pertinence given that, for facial reviewers, professional experience has been demonstrated to bear no correlation to face-matching accuracy (White, Kemp, Jenkins, Matheson, et al., 2014) and in the majority of studies reviewers perform at comparable levels to controls (White et al., 2021).

Almost all agencies included face-matching exercises of some form in their training, barring one reviewer and one examiner training programme. Of those that do, not all provided feedback on match responses, despite empirical evidence that feedback on face matching can improve accuracy (White, Kemp, Jenkins, \& Burton, 2014). Feedback on representative tasks has been demonstrated as a key contributor to developing perceptual expertise (Edmond et al., 2017).

Training approaches that encourage the use of specific facial features were more common in examiner training and significantly more examiner training programmes included the use of 
facial feature checklists. Facial feature comparison (Megreya \& Bindemann, 2018) and feature checklists (Towler et al., 2017) have both been demonstrated to improve accuracy on matching face pairs, with the analysis and comparison of features being the recommended approach for face matching by practitioner working groups, termed the morphological approach. The greater adoption of facial feature comparison and feature checklists may be a contributor to the enhanced accuracy of examiners at the group level.

The variable adoption of evidence-based approaches in face-matching training, with some of the lowest adoption rates observed in the entire survey, suggests that there is a disconnect between face-matching research and professional training practices. Therefore, greater communication and collaboration is required between face-matching researchers and practitioner working groups that develop training guidelines, to ensure more widespread adoption of evidence-based approaches that have been empirically proven to improve facematching accuracy.

\section{General Discussion}

To date there has been no longitudinal study of face-matching training to validate the effectiveness of longer training programmes. Only short professional training courses of up to three days have been empirically tested (Towler et al., 2019) and have been shown to have little, if any impact on improving face-matching accuracy. For the agencies that took part in the survey all examiner training and almost half of reviewer training is at least one week in duration, with most reviewers trained for one to six months and most examiners for one to five years. Searston \& Tangen (2017a) tracked the performance of 24 fingerprint trainees over the period of one year, with tests measuring four aspects of fingerprint expertise delivered every three months. Using a composite score of the four measures, they found the most significant gains in expertise to occur in the first three months with gains steadily plateauing after this 
point. Future research should investigate whether face-matching expertise shows a similar emergence over time and determine if longer durations of training could be the source of enhanced examiner performance.

Searston \& Tangen (2017a) also found that the initial perceptual expertise of trainees at fingerprint matching prior to training was a reliable predictor of ongoing performance throughout the training programme. This means that for fingerprint expertise, pre-screening of applicants appears effective in identifying high performers who will continue to develop their enhanced expertise through training. Pre-screening using face-matching tests has been widely advocated for identifying high performing individuals for applied roles (Bobak et al., 2016; Ramon, Bobak, \& White, 2019; White, Dunn, et al., 2015), but to date no study has investigated the interaction between pe-screening and long term training in applied face matching. According to the survey only $40 \%$ of agencies are pre-screening the face-matching abilities of reviewers and examiners prior to training. If the development of fingerprint-matching expertise is analogous to face-matching expertise, then pre-screening may be useful tool in evaluating the effectiveness of longer-term training and identifying the top performers for subsequent enrolment into training.

Studies of face-matching training have found short online and instructor driven courses to be largely ineffective at improving face-matching ability. However, from the survey results it appears agencies are using a range of different training delivery techniques for reviewer and examiner training, including independent learning and one-to-one mentoring. In-house mentoring is seen to be an important part of professional development across the forensic sciences (Ashcroft, Daniels, \& Hart, 2004) and practitioner working groups in face matching recommend that facial reviewers and examiners be assigned a workplace mentor during their training and development (European Network of Forensic Science Institutes, 2018; Facial 
Identification Scientific Working Group, 2019c). As yet no study has evaluated the effectiveness of workplace mentoring in developing face-matching expertise. As recommended by Towler et al. (2019), mentoring in face matching should also be investigated as a part of the evaluation of longitudinal face-matching training.

Researchers also advocate the use of evidence-based training practices to develop perceptual expertise (Searston \& Tangen, 2017; Towler et al., 2019). The results from the survey demonstrate that the use of evidence-based training practices is relatively low for both examiner and reviewer training. Whilst training guidelines for face-matching practitioners do exist (e.g. Facial Identification Scientific Working Group, 2010), these guidelines are prescriptive on the topics that a training course should include but are lacking details on how to design evidence-based training courses that develop and measure emerging perceptual expertise over time. In this regard, there needs to be greater collaboration between the practitioner community and relevant researchers in human-performance testing, cognitive science and psychology to design empirically derived face-matching training that is proven to develop expertise.

The survey results have revealed a general trend where examiners are trained for longer than reviewers, via a more diverse range of delivery methods and trained using a wider range of topics and research-based approaches. These observations may provide an explanation for the enhanced face-matching accuracy of examiners at the group level. Although there are overall differences in duration, delivery techniques and topics between reviewer and examiner training at the group level, there are also substantial individual differences in training practices within each group. Perhaps the most notable difference within both reviewer and examiner training is duration. Reviewer training ranged from less than a day to up to 12 months. $40 \%$ of agencies run reviewer training for five days or less, this is particularly alarming given that training 
courses of three days and less have been demonstrated to provide no consistent improvement in face-matching accuracy (Towler et al., 2019). In the upper range of reviewer training duration, $40 \%$ of agencies provided one to six months of training. Likewise, for examiners training durations ranged from one to four weeks to up to five plus years. Differences in training practices were also observed for delivery methods, topics and inclusion of research-based exercises. This diversity in results indicates a lack of standardisation in training practices between different agencies and may be a contributing factor towards the individual differences in reviewer and examiner accuracy observed in the literature (White et al., 2021).

In addition to longitudinal training studies and the development of evidence-based training practices, the practitioner and research communities should also move towards white box testing of examiners and reviewers. In white box testing participants disclose information about the processes and procedures they used to complete the test. White box testing has been used to better understand the expertise of forensic fingerprint examiners (e.g. Ulery, Hicklin, Roberts, \& Buscaglia, 2015). In a white box testing scenario, agencies could report how their personnel are selected and trained. By disclosing the extent of training that reviewers and examiners have received in a white box face-matching test, relationships between different training approaches and face-matching performance may be found. In this manner it may be possible to identify agencies with effective training regimes and use these as a model for the wider face-matching community, which could in turn reduce the wide range in individual differences within these practitioner communities and mitigate against high risk errors in applied face-matching scenarios. 


\section{References}

Alenezi, H. M., \& Bindemann, M. (2013). The Effect of Feedback on Face-Matching Accuracy. Applied Cognitive Psychology, 27, 735-753.

Ashcroft, J., Daniels, D. J., \& Hart, S. V. (2004). Education and Training in Forensic Science: A Guide for Forensic Science Laboratories, Educational Institutions, and Students.

Bobak, A. K., Dowsett, A. J., \& Bate, S. (2016). Solving the border control problem: Evidence of enhanced face matching in individuals with extraordinary face recognition skills. PLoS ONE, 11(2). https://doi.org/10.1371/journal.pone.0148148

Burton, M., Wilson, S., Cowan, M., \& Bruce, V. (1999). Face Recognition in Poor-Quality Video: Evidence from Security Surveillance. Psychological Science, 10(3), 243-248. https://doi.org/10.1111/1467-9280.00144

Dowsett, A. J., \& Burton, A. M. (2015). Unfamiliar face matching: Pairs out-perform individuals and provide a route to training. British Journal of Psychology, 106(3), 433445. https://doi.org/10.1111/bjop.12103

Edmond, G., Biber, K., Kemp, R., \& Porter, G. (2009). Law’s Looking Glass: Expert Identification Evidence Derived from Photographic and Video Images. Current Issues in Criminal Justice, 20(3), 337-377.

Edmond, G., Towler, A., Growns, B., Ribeiro, G., Found, B., White, D., ... Martire, K. (2017). Thinking forensics: Cognitive science for forensic practitioners. Science \& Justice, 57(2), 144-154. https://doi.org/10.1016/j.scijus.2016.11.005

European Network of Forensic Science Institutes. (2015). ENFSI Best Practice Manual for Fingerprint Examination (Vol. 01). Retrieved from http://enfsi.eu/wpcontent/uploads/2016/09/6._fingerprint_examination_0.pdf

European Network of Forensic Science Institutes. (2018). ENFSI Best Practice Manual for Facial Image Comparison (Vol. 01). Retrieved from http://enfsi.eu/wpcontent/uploads/2017/06/ENFSI-BPM-DI-01.pdf

Facial Identification Scientific Working Group. (2010). Guidelines and Recommendations for 
Facial Comparison Training to Competency. Facial Identification Scientific Working Group. Retrieved from https://fiswg.org/FISWG_Training_Guidelines_Recommendations_v1.1_2010_11_18.p df

Facial Identification Scientific Working Group. (2012). Guidelines for Facial Comparison Methods.

Facial Identification Scientific Working Group. (2019a). Guide for Facial Comparison Training of Examiners to Competency.

Facial Identification Scientific Working Group. (2019b). Guide for Facial Comparison Training of Reviewers to Competency.

Facial Identification Scientific Working Group. (2019c). Guide for Mentorship of Facial Comparison Trainees in Role Based Facial Comparison. Retrieved from https://fiswg.org/FISWG_Mentorship_for_Facial_Comparison_Trainees_v1.0_2019051 $0 . p d f$

Houlton, T. M. R., \& Steyn, M. (2018). Finding Makhubu: A morphological forensic facial comparison. Forensic Science International, 285(February), 13-20. https://doi.org/10.1016/j.forsciint.2018.01.022

Kleinberg, K. F., \& Vanezis, P. (2007). Variation in proportion indices and angles between selected facial landmarks with rotation in the Frankfort plane. Medicine, Science, and the Law, 47(2), 107-116. https://doi.org/10.1258/rsmmsl.47.2.107

Lander, K., Bruce, V., \& Bindemann, M. (2018). Use-inspired basic research on individual differences in face identification: implications for criminal investigation and security. Cognitive Research: Principles and Implications, 3(1), 26. https://doi.org/10.1186/s41235-018-0115-6

Langdon, F. J. (2014). Evidence of mentor learning and development: an analysis of New Zealand mentor/mentee professional conversations. Professional Development in Education, 40(1), 36-55. https://doi.org/10.1080/19415257.2013.833131

Mallett, X., \& Evison, M. P. (2013). Forensic facial comparison: Issues of admissibility in the 
development of novel analytical technique. Journal of Forensic Sciences, 58(4), 859865. https://doi.org/10.1111/1556-4029.12127

Mayfield, M. (2010). Tacit knowledge sharing: Techniques for putting a powerful tool in practice. Development and Learning in Organisations, 24(1), 24-26. https://doi.org/10.1108/14777281011010497

McNeill, M. A., Suchomska, M., \& Strathie, A. (2015). Expert facial comparison evidence: Science versus pseudo science. Psychology and Law, 5(4), 127-140. https://doi.org/10.17759/psylaw.2015050411

Megreya, A. M., \& Bindemann, M. (2018). Feature instructions improve face-matching accuracy. PLoS ONE, 13(3), 1-16. https://doi.org/10.1371/journal.pone.0193455

Moreton, R., \& Morley, J. (2011). Investigation into the use of photoanthropometry in facial image comparison. Forensic Science International, 212(1-3), 231-237. https://doi.org/10.1016/j.forsciint.2011.06.023

Norell, K., Läthén, K. B., Bergström, P., Rice, A., Natu, V., \& O’Toole, A. (2015). The Effect of Image Quality and Forensic Expertise in Facial Image Comparisons. Journal of Forensic Sciences, 60(2), 331-340. https://doi.org/10.1111/1556-4029.12660

Noyes, E. (2016). Face Recognition in Challenging Situations.

Phillips, P. J., Yates, A. N., Hu, Y., Hahn, C. A., Noyes, E., Jackson, K., ... O’Toole, A. J. (2018). Face recognition accuracy of forensic examiners, superrecognizers, and face recognition algorithms. Proceedings of the National Academy of Sciences, 201721355. https://doi.org/10.1073/pnas.1721355115

Ramon, M., Bobak, A. K., \& White, D. (2019). Super-recognizers: From the lab to the world and back again. British Journal of Psychology, 110(3), 461-479.

https://doi.org/10.1111/bjop.12368

Ritz-Timme, S., Gabriel, P., Obertovà, Z., Boguslawski, M., Mayer, F., Drabik, A., ... Cattaneo, C. (2011). A new atlas for the evaluation of facial features: Advantages, limits, and applicability. International Journal of Legal Medicine, 125(2), 301-306. https://doi.org/10.1007/s00414-010-0446-4 
Searston, R. A., \& Tangen, J. M. (2017). The Emergence of Perceptual Expertise with Fingerprints Over Time. Journal of Applied Research in Memory and Cognition, 6(4), 442-451. https://doi.org/10.1016/j.jarmac.2017.08.006

Sosik, J. J., Godshalk, V. M., \& Yammarino, F. J. (2004). Transformational leadership, learning goal orientation, and expectations for career success in mentor-protégé relationships: A multiple levels of analysis perspective. Leadership Quarterly, 15(2), 241-261. https://doi.org/10.1016/j.leaqua.2004.02.003

Strathie, A., \& McNeill, A. (2016). Facial Wipes don't Wash: Facial Image Comparison by Video Superimposition Reduces the Accuracy of Face Matching Decisions. Applied Cognitive Psychology, 30(4), 504-513. https://doi.org/10.1002/acp.3218

Strathie, A., Mcneill, A., \& White, D. (2012). In the Dock: Chimeric Image Composites Reduce Identification Accuracy. Applied Cognitive Psychology, 26(1), 140-148. https://doi.org/10.1002/acp.1806

Towler, A. (2016). Match me if you can : Evaluating professional training for facial image comparison. University of New South Wales.

Towler, A., Kemp, R. I., Mike Burton, A., Dunn, J. D., Wayne, T., Moreton, R., \& White, D. (2019). Do professional facial image comparison training courses work? PLoS ONE, 14(2), 1-17. https://doi.org/10.1371/journal.pone.0211037

Towler, A., White, D., \& Kemp, R. I. (2014). Evaluating training methods for facial image comparison: The face shape strategy does not work. Perception, 43(2-3), 214-218. https://doi.org/10.1068/p7676

Towler, A., White, D., \& Kemp, R. I. (2017). Evaluating the feature comparison strategy for forensic face identification. Journal of Experimental Psychology: Applied, 23(1), 47-58. https://doi.org/10.1037/xap0000108

Ulery, B. T., Hicklin, R. A., Roberts, M. A., \& Buscaglia, J. A. (2015). Changes in latent fingerprint examiners' markup between analysis and comparison. Forensic Science International, 247(1), 54-61. https://doi.org/10.1016/j.forsciint.2014.11.021

White, D., Dunn, J. D., Schmid, A. C., \& Kemp, R. I. (2015). Error rates in users of 
automatic face recognition software. PLoS ONE, 10(10).

https://doi.org/10.1371/journal.pone.0139827

White, D., Kemp, R. I., Jenkins, R., \& Burton, A. M. (2014). Feedback training for facial image comparison. Psychonomic Bulletin \{\&\} Review, 21(1), 100-106. https://doi.org/10.3758/s13423-013-0475-3

White, D., Kemp, R. I., Jenkins, R., Matheson, M., \& Burton, A. M. (2014). Passport officers' errors in face matching. PLoS ONE, 9(8). https://doi.org/10.1371/journal.pone.0103510

White, D., Phillips, P. J., Hahn, C. A., Hill, M., \& O’Toole, A. J. (2015). Perceptual expertise in forensic facial image comparison. Proceedings of the Royal Society B: Biological Sciences, 282(1814), 20151292. https://doi.org/10.1098/rspb.2015.1292

White, D., Towler, A., \& Kemp, R. I. (2021). Understanding professional expertise in unfamiliar face matching. In M. Bindemann (Ed.), Forensic Face Matching: Research and practice. Oxford University Press.

Wirth, B. E., \& Carbon, C.-C. (2017). An easy game for frauds? Effects of professional experience and time pressure on passport-matching performance. Journal of Experimental Psychology: Applied, 23(2), 138-157. https://doi.org/10.1037/xap0000114

Woodhead, M. M., Baddeley, a. D., \& Simmonds, D. C. V. (1979). On Training People to Recognize Faces. Ergonomics, 22(3), 333-343. https://doi.org/10.1080/00140137908924617 